\title{
Local Control Theory in Trajectory Surface Hopping Dynamics Applied to the Excited-State Proton Transfer of 4-Hydroxyacridine
}

\author{
Basile F. E. Curchod, ${ }^{[b]}$ Thomas J. Penfold ${ }^{*[c]}$ Ursula Rothlisberger, $^{[a]}$ and Ivano Tavernelli*[a]
}

The application of local control theory combined with nonadiabatic ab initio molecular dynamics to study the photoinduced intramolecular proton transfer reaction in 4-hydroxyacridine was investigated. All calculations were performed within the framework of linear-response time-dependent density functional theory. The computed pulses revealed important information about the underlying excited-state nuclear dynamics highlighting the involvement of collective vibrational modes that would normally be neglected in a study performed on model systems constrained to a subset of the full configuration space. This study emphasizes the strengths of local contro theory for the design of pulses that can trigger chemical reactions associated with the population of a given molecular excited state. In addition, analysis of the generated pulses can help to shed new light on the photophysics and photochemistry of complex molecular systems.

\section{Introduction}

The aim of quantum control is to manipulate the dynamics of a nuclear wavepacket by modulating the amplitude, frequencies, and phases of a laser pulse used to excite a system. Owing to the development of laser technologies, this has been achieved up to a certain point ${ }^{[1-3]}$ and is principally realized by using a closed loop-learning algorithm. However, while control of the quantum dynamics can be achieved, this is often done without a close understanding of the underlying molecular mechanism. Until recently, most quantum control experiments and simulations were performed on isolated atoms or molecules in the gas phase. ${ }^{[4-8]}$ However, this field has recently been extended to a wider variety of problems such as, for example, the manipulation of coherent phonons in solids, ${ }^{[9]}$ energy flow and isomerization processes in biomolecules, ${ }^{[10-12]}$ discrimination of molecules, ${ }^{[13-15]}$ and bond formations on catalytic surfa-

[a] Prof. Dr. U. Rothlisberger, Dr. I. Tavernelli ${ }^{+}$

Laboratory of Computational Chemistry and Biochemistry

Ecole Polytechnique Fédérale de Lausanne

1015 Lausanne (Switzerland)

E-mail:ita@zurich.ibm.com

[b] Dr. B. F. E. Curchod ${ }^{++}$

Laboratory of Computational Chemistry and Biochemistry

Ecole Polytechnique Fédérale de Lausanne

1015 Lausanne (Switzerland)

[c] Dr. T. J. Penfold

SwissFEL, Paul Scherrer Inst.

5232 Villigen (Switzerland)

E-mail: thomas.penfold@psi.ch

$\left.{ }^{+}\right]$Current address:

IBM Research GmbH, Zurich Research Laboratory

8803 Rüschlikon (Switzerland)

$\left.{ }^{[++}\right]$Current address:

Department of Chemistry, Stanford University

Stanford, California 94305 (USA)

Supporting Information for this article is available on the WWW under http://dx.doi.org/10.1002/cphc.201500190. ces. ${ }^{[16]}$ In addition, the concepts of quantum control have been applied to nonlinear spectroscopy and microscopy. ${ }^{[17]}$ In this last case, shaped femtosecond pulses have been used to enhance resolution (e.g. by controlling off-diagonal features) ${ }^{[18]}$ and to improve detection. On even shorter timescales, attosecond spectroscopy can potentially be used to directly control the electron dynamics in molecules. ${ }^{[19]}$

To understand the underlying mechanisms of the control dynamics and/or the effects of a specific shaped laser pulse, theoretical simulations are often important. A variety of different theoretical methods have been developed to perform control simulations ${ }^{[20-22]}$ or to rationalize experimental pulse-shaping results. ${ }^{[23]}$ Among them, optimal control theory has been the most widely used. In this approach, a pulse is optimized through variation of a properly defined functional ${ }^{[24]}$ or by means of a learning algorithm in analogy with the experimental optimization procedures. ${ }^{[7]}$ This approach has been implemented almost exclusively within the framework of quantum wavepacket simulations, and therefore, the computational expense of solving the time-dependent Schrödinger equation many times and the requirement of having precalculated potential energy surfaces (PESs) has limited its applications to model systems with a maximum of four degrees of freedom (DOF). Although such methods are usually sufficient for the study of small molecules predominantly addressed thus far by coherent control experiments, they are not generally suitable to address the dynamics of larger systems, such as biomolecules or solids.

To study the effect of shaped laser pulses for larger systems in an explicit environment, the most feasible and attractive approach is mixed quantum/classical dynamics. Indeed, the coupling of such approaches with external time-dependent electric fields has a long tradition. ${ }^{[25,26]}$ Of particular interest for the simulation of realistic systems in their full, unconstrained con- 
figuration space are quantum/classical approaches based on on-the-fly techniques such as trajectory surface hopping (TSH), ${ }^{[27]}$ where all necessary quantities required for the nuclear dynamics are computed locally at a given nuclear configuration. Several electronic structure methods have so far been successfully coupled with TSH, among which the most commonly used are ab initio ${ }^{[28,29]}$ and semiempirical methods ${ }^{[30,31]}$ and linear-response time-dependent density functional theory (LR-TDDFT). ${ }^{[32,33]}$ Recent extensions of TSH to include arbitrary coupling terms have also been proposed. ${ }^{[34-46]}$ In particular, a LR-TDDFT-based TSH ab initio molecular dynamics scheme that couples the electronic and nuclear dynamics to external time-dependent electric fields ${ }^{[37]}$ was recently extended to allow for the "on-the-fly" design of laser pulses with desired properties, including, for instance, the ability to selectively populate a target excited state by using local control theory (LCT). ${ }^{[38]}$ LCT coupled with TSH ab initio molecular dynamics, ${ }^{[38]}$ TSH/LCT, offers a powerful alternative to the more sophisticated optimal control algorithm. In the standard LCT approach, a target is defined and a control pulse that ensures the increase (or decrease) of the target expectation value is computed on-the-fly by using exclusively instantaneous electronic structure quantities evaluated at each time step, which avoids the need for forward/backward iterations. Despite its simplicity, LCT can be successfully applied whenever the optimization process deals with only a few observables at a time..$^{[39-41]}$ In particular, as only a single time-propagation is required, LCT is particularly attractive in combination with nonadiabatic ab initio molecular dynamics for the treatment of medium to large sized molecules in their full configuration space.

In this work, we adopt a particular variant of LCT, in which the desired target quantity is the selective population of a given excited state. ${ }^{[38]}$ Starting from a system evolving in its ground state, the designed TSH/LCT pulse will transfer amplitude to the desired electronic state eventually to lead to a hop of the (classical) trajectory into the target state: a TSH realization of the photoabsorption process. The effect of the designed pulse will therefore be to drive the dynamics from an initial electronic state (in most cases the ground state) to a selected excited-state PES, on which new chemical processes can be observed. However, it is important to stress that in this way the external field only influences the nuclear dynamics indirectly, as the nuclear wavefunction does not enter, at any time, in the pulse-shaping process. As a consequence, TSH/LCT pulses do not directly drive the nuclear degrees of freedom, which only react to the change in PES induced by the surface hop.

Finally, this flavor of TSH/LCT, which targets exclusively electronic state populations, can also be employed as an ab initio technique to start nonadiabatic molecular dynamics calculations on a desired excited state without performing an ad hoc promotion of initial ground-state configurations in a desired excited electronic state.

As an application of our LCT approach based on TSH in the framework of LR-TDDFT, we present results on the ultrafast photochemical intramolecular proton-transfer reaction in the molecular system 4-hydroxyacridine $(4-\mathrm{HA}) \cdot{ }^{[42,43]}$ This process has already been studied both experimentally and theoretical- ly, however, in the latter case, by static calculations only. ${ }^{[42,43]}$ These studies have reported that in the ground state the intramolecular proton transfer is hindered by a prohibitively high energy barrier that is highly reduced if the molecule is excited into its first excited state $\left(S_{1}\right)$. (With the presence of a bridging water molecule between the donor and the acceptor site, the proton transfer is even expected to become barrierless. ${ }^{[43]}$ ) This is made possible by the electronic character of the $S_{1}$ state, which-at the ground-state geometry-is predominantly assigned to a HOMO-LUMO transition ( $88.5 \%$, see Figure 1) with an enhanced acidity of the hydroxyl group and a stronger basicity of the nitrogen atom in the acridine ring. ${ }^{[42,43]}$

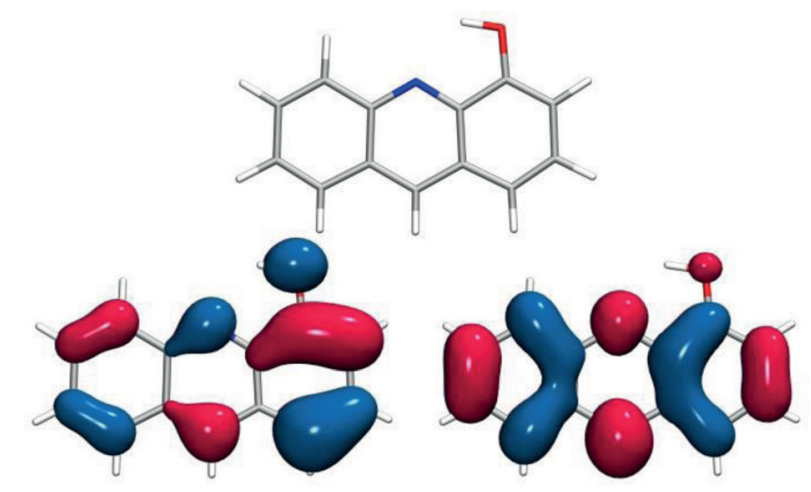

Figure 1. Top) 4-Hydroxyacridine (4-HA) (in the xy plane). Bottom) KohnSham HOMO (left) and LUMO (right) of 4-HA at the DFT/PBE level of theory. Orbitals are plotted with an isovalue of 0.03 .

\section{Theory}

Tully's fewest switches trajectory surface hopping $(\mathrm{TSH})^{[27]} d y-$ namics can easily be coupled to external electromagnetic fields, as shown in a number of recent publications. ${ }^{[25,34-37]}$ Within this approach, the molecule of interest is first prepared in its ground state. Through coupling with an external electromagnetic field $\mathbf{E}(t)$, the amplitude is transferred to an excited state depending on the size of the associated transition dipole moment $\boldsymbol{\mu}_{j i}\left(\mathbf{R}^{[\alpha]}\right)$. The corresponding modified TSH equation for the amplitude $C_{j}^{[\alpha]}$ associated to an electronic state $j$ and a trajectory $\alpha$ is [Eq. (1)]:

$$
\begin{aligned}
& i \hbar \dot{C}_{j}^{[\alpha]}(t)= \\
& \sum_{i}^{\infty} C_{i}^{[\alpha]}(t)\left(E_{j}^{\mathrm{el}}\left(\mathbf{R}^{[\alpha]}\right) \delta_{j i}-i \hbar \dot{\mathbf{R}}^{[\alpha]} \cdot \mathbf{d}_{j i}\left(\mathbf{R}^{[\alpha]}\right)-\mathbf{E}(t) \cdot \boldsymbol{\mu}_{j i}\left(\mathbf{R}^{[\alpha]}\right)\right)
\end{aligned}
$$

In Equation (1), $E_{j}^{\mathrm{el}}(\mathbf{R})$ is defined as [Eq. (2)]

$E_{j}^{\mathrm{el}}(\mathbf{R})=\int d \mathbf{r} \Phi_{j}^{*}(\mathbf{r} ; \mathbf{R}) \hat{H}_{\mathrm{el}} \Phi_{j}(\mathbf{r} ; \mathbf{R})$

and are the matrix elements of the electronic Hamiltonian $\hat{H}_{\mathrm{el}}$ in the set of basis functions $\{\Phi .(\mathbf{r} ; \mathbf{R})\}$ that are solutions to the electronic time-independent Schrödinger equation for fixed nuclear coordinates $\mathbf{R}$ ( $\mathbf{r}$ represents the collective vector of all 
electrons in the system). The nonadiabatic coupling vectors between two electronic states $j$ and $i$ are given by [Eq. (3)]:

$\mathbf{d}_{j i}(\mathbf{R})=\int d \mathbf{r} \Phi_{j}^{*}(\mathbf{r} ; \mathbf{R}) \nabla_{\mathbf{R}} \Phi_{i}(\mathbf{r} ; \mathbf{R})$

and $\dot{\mathbf{R}}^{[\alpha]}$ in Equation (1) is a collective notation to denote the velocity of the classical nuclei for the trajectory $\alpha$. For more details on LR-TDDFT-based TSH dynamics, the reader is referred to recent literature on the subject. ${ }^{[44,45]}$

In the following, pulse shaping is achieved through LCT. ${ }^{[40]}$ As previously mentioned, the aim of this approach is to design, on-the-fly, the electric field that ensures an increase (or decrease) in some predefined expectation values such as the population of a target excited state.

Starting from the time dependence of the expectation value of an arbitrary operator $\hat{B}$ governed by the total Hamiltonian $\hat{H}=\hat{H}_{\text {mol }}+\hat{H}_{\text {int }}$ [Eq. (4)]:

$$
\begin{aligned}
\frac{d\langle\hat{B}\rangle(t)}{d t}= & \frac{i}{\hbar} \iint d \mathbf{r} d \mathbf{R} \Psi^{*}(\mathbf{r}, \mathbf{R}, t)\left[\hat{H}_{\mathrm{mol}}, \hat{B}\right] \Psi(\mathbf{r}, \mathbf{R}, t) \\
& +\frac{i}{\hbar} \iint d \mathbf{r} d \mathbf{R} \Psi^{*}(\mathbf{r}, \mathbf{R}, t)\left[\hat{H}_{\mathrm{int}}, \hat{B}\right] \Psi(\mathbf{r}, \mathbf{R}, t)
\end{aligned}
$$

(in which $\hat{H}_{\text {mol }}$ is the molecular Hamiltonian and $\hat{H}_{\text {int }}=-\hat{\mu} \mathbf{E}(t)$ is the matter-field interaction Hamiltonian) and by assuming that $\hat{H}_{\text {mol }}$ commutes with $\hat{B}$ (which is only true in the absence of nonadiabatic couplings) we obtain [Eq. (5)]:

$$
\frac{d\langle\hat{B}\rangle(t)}{d t}=-\mathbf{E}(t) \frac{i}{\hbar} \iint d \mathbf{r} d \mathbf{R} \Psi^{*}(\mathbf{r}, \mathbf{R}, t)[\hat{\mu}, \hat{B}] \Psi(\mathbf{r}, \mathbf{R}, t)
$$

Note that in the presence of nonadiabatic couplings Equation (5) will contain an additional time-dependent term (see refs. $[41,46,47])$. To control the population of a particular electronic state $\left|\Phi_{i}\right\rangle$, we introduce the projector operator $\hat{P}_{i}=\left|\Phi_{i}\right\rangle\left\langle\Phi_{i}\right|$. The time evolution of the state population is simply given by [Eq. (6)]:

$$
\frac{d\left\langle\hat{P}_{i}\right\rangle(t)}{d t}=-\mathbf{E}(t) \frac{i}{\hbar} \iint d \mathbf{r} \mathbf{R} \mathbf{R} \Psi^{*}(\mathbf{r}, \mathbf{R}, t)\left[\hat{\mu}, \hat{P}_{i}\right] \Psi(\mathbf{r}, \mathbf{R}, t)
$$

Using the TSH ansatz for a classical trajectory $\alpha$ [Eq. (7)]:

$$
\Psi^{[\alpha]}(\mathbf{r}, \mathbf{R}, t)=\sum_{j}^{\infty} C_{j}^{[\alpha]}(t) \Phi_{j}(\mathbf{r} ; \mathbf{R})
$$

and the corresponding representation of the nuclear density by a delta function, Equation (6) becomes [Eq. (8)]:

$$
\frac{d\left\langle\hat{P}_{i}^{[\alpha]}\right\rangle(t)}{d t}=-\frac{2}{\hbar} \mathbf{E}^{[\alpha]}(t) \sum_{j}^{\infty} \Im\left[C_{i}^{[\alpha]}{ }^{*}(t) \boldsymbol{\mu}_{i j}\left(\mathbf{R}^{[\alpha]}\right) C_{j}^{[\alpha]}(t)\right]
$$

with $\boldsymbol{\mu}_{i j}\left(\mathbf{R}^{[\alpha]}\right)=-e \sum_{k}^{N_{\mathrm{el}}} \int d \mathbf{r} \Phi_{i}^{*}\left(\mathbf{r} ; \mathbf{R}^{[\alpha]}\right) \hat{\mathbf{r}}_{k} \Phi_{j}\left(\mathbf{r} ; \mathbf{R}^{[\alpha]}\right)$. From inspection of Equation (8) it becomes evident that the electric field [Eq. (9)]:
$\mathbf{E}^{[\alpha]}(t)= \pm \lambda \sum_{j}^{\infty} \Im\left[C_{i}^{[\alpha]}{ }^{*}(t) \boldsymbol{\mu}_{i j}\left(\mathbf{R}^{[\alpha]}\right) C_{j}^{[\alpha]}(t)\right]$

will ensure that $\left\langle\hat{P}_{i}^{[\alpha]}\right\rangle(t)$ increases (minus sign) or decreases (plus sign) at all times. We note that modification of the TSH algorithm proposed in this work is valid as long as the generated pulse is not too strong, that is, that it does not trigger an excessively large number of hops during the dynamics or that it does not strongly alter the shape of the adiabatic PESs. ${ }^{[48]}$ It is important to stress that all approximations related to the use of the TSH ansatz for nonadiabatic dynamics with classical trajectories (e.g. see ref. [49]) will also impact the quality of the computed LCT pulse. For example, the missing description of decoherence inherent to the standard TSH algorithm can play a role in the pulse-shaping process, as noted in ref. [38] and further stressed in recent work on field-coupled TSH dynamics. ${ }^{[50]}$

In summary, if a targeted chemical process can be associated to a well-defined excited-state transition, local control theory coupled to TSH dynamics offers a potentially valuable tool for the optimization of the external time-dependent electric field able to efficiently trigger the desired reaction, as it will be discussed in the next sections for the case of the intramolecular proton transfer in 4-HA.

\section{System Setup and Computational Details}

We consider an isolated 4-HA molecule placed in a simulation box of dimensions $16 \times 16 \times 10 \AA$. If not specified differently, the calculations were performed by using the CPMD package, ${ }^{[51]}$ by employing Martins-Troullier-type pseudopotentials, $^{[52]}$ a cutoff of 100 Ry for the plane wave basis set, and a convergence threshold of $10^{-7}$ au for the wavefunction. The ground state (GS) and the first three excited states $\left(S_{1}, S_{2}\right.$, and $\mathrm{S}_{3}$ ) were included in the calculations, and the LR-TDDFT equations were solved within the Tamm-Dancoff approximation (TDA) to obtain all excitation energies and nuclear forces. ${ }^{[33]}$ The Perdew-Burke-Ernzerhof (PBE) $x c$ functional ${ }^{[54]}$ was used, together with the adiabatic approximation for the corresponding $x c$ kernel. The quality of the PBE functional for the excitedstate dynamics was assessed by comparison with energies obtained by using LR-TDDFT with the hybrid functional PBE0 ${ }^{[55]}$ on geometries sampled along a selected PBE TSH/LCT trajectory (see the Supporting Information). In the latter case, the energies of the electronic states visited during the nonadiabatic dynamics mainly showed a rigid shift relative to the ground state that depended on the amount of exact exchange, and this left the energy differences between the excited states essentially unperturbed. The molecule was equilibrated at $300 \mathrm{~K}$ by using ground-state Born-Oppenheimer molecular dynamics, as implemented in CPMD. ${ }^{[51]}$ From the equilibrated groundstate Boltzmann distribution, different initial configurations were randomly selected and used as starting geometries for the nonadiabatic dynamics. Initial velocities were set equal to the corresponding ground-state values. 
For the TSH dynamics with the $\Pi$ pulse, the vector potential $\mathbf{A}(t)$ is given by [Eq. (10)]:

$\mathbf{A}(t)=-A_{0} \boldsymbol{\varepsilon}^{\lambda} \exp \left(-\frac{\left(t-t_{0}\right)^{2}}{T^{2}}\right) \sin (\omega t)$

with a frequency $\omega$ centered at the value of the energy gap $E_{\mathrm{S}_{1}}^{\mathrm{el}}-E_{\mathrm{GS}}^{\mathrm{el}}=2.55 \mathrm{eV}$ computed at the ground-state optimized geometry, and $A_{0} / c=0.1067, t=2000 \mathrm{au}$, and $T=800 \mathrm{au}$.

The TSH/LCT calculation was initiated by a seeding phase of $2.4 \mathrm{fs}$, during which an external field of 0.005 au was used to promote an infinitesimal starting population into the target state. This was necessary to initiate the LCT dynamics, which remained ineffective as long as the target state had exactly zero population according to Equation (9). The rest of the TSH/ LCT dynamics was performed with a coupling strength $\lambda$ of 0.1 with an external electric field polarized along the $y$ axis (see Figure 1 for the orientation of 4-HA). As a reference, we note that, at the optimized ground-state geometry, the following transition dipole moments were observed: $\mu_{\mathrm{GS} / \mathrm{S}_{1}}^{y}=0.398 \mathrm{au}$, $\mu_{\mathrm{GS} / \mathrm{S}_{2}}^{y}=-0.084 \mathrm{au}$, and $\mu_{\mathrm{GS} / \mathrm{S}_{3}}^{y}=0.235 \mathrm{au}$. The nonadiabatic dynamics was initiated in the electronic molecular ground state, and at each nuclear time step the field derived in Equation (9) was constructed and applied to the dynamics of the amplitudes according to Equation (1). The time step for the integration of the nuclear degrees of freedom was set to $1 \mathrm{au}$. We only considered the transition dipole moments between the GS and the excited states, and consequently, the summation in Equation (9) was restricted to $j=0$. This implies that the population of the target state $S_{1}$ could only originate from depopulation of the ground state. This approximation is validated by the fact that only weak amplitude transfer between GS and $S_{2}$ or $\mathrm{S}_{3}$ was observed along the dynamics (see Figure 3, discussed later in the text). The final pulse is therefore expected to be essentially unaffected by this approximation. A complete account of the implementation of LCT theory in the framework of LRTDDFT-based TSH dynamics in the CPMD code ${ }^{[51]}$ is given in refs. $[38,44]$.

Frequency-resolved optical grating (FROG) analysis of the computed LCT pulses was performed by using the XFROG utility distributed within the Heidelberg MCTDH package. ${ }^{[56,57]}$ All molecular representations were produced with $\mathrm{VMD}^{[58]}$ version 1.9.1.

\section{Results and Discussion}

We first discuss the dynamics of 4-HA under the influence of a $\Pi$ pulse parametrized according to Equation (10) and depicted in the bottom panel of Figure 2. During the first $50 \mathrm{fs}$ of the dynamics, population is smoothly transferred between GS and $S_{1}$. However, the maximum of $\left|C_{S_{1}}(t)\right|^{2}$ never reaches a value higher than $42 \%$, and the trajectory hops to the target state $S_{1}$ for only a short period of time at approximately $t \sim$ $77 \mathrm{fs}$. In conclusion, this rather weak $\Pi$ pulse $\left(E_{y}^{\max }=0.01 \mathrm{au}\right)$ is unable to efficiently promote the selective population of $S_{1}$ and rather leads to a mixing of the population of the GS and

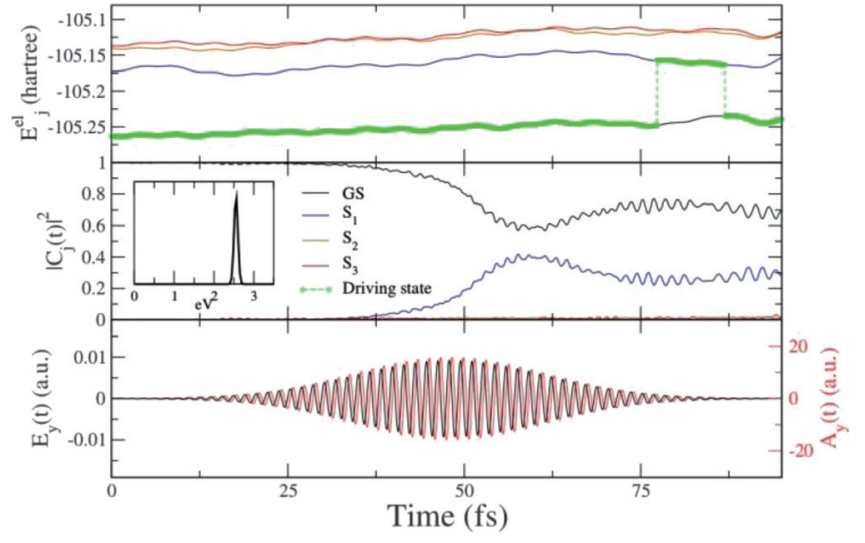

Figure 2. Dynamics of 4-HA under the action of a polarized $\Pi$ pulse with central frequency $\omega=2.55 \mathrm{eV}$. Top panel) Time series of the potential energy curves obtained with DFT/PBE and LR-TDDFT/PBE/TDA calculations. Color code: GS (black), $S_{1}$ (blue), $S_{2}$ (orange) and $S_{3}$ (red). The green line with circles highlights the force (driving) state. Middle panel) Probability $\left(\left|C_{j}(t)\right|^{2}\right)$ of each state for one trajectory. The inset shows the Fourier transform of the entire $\Pi$ pulse. Bottom panel) Applied vector potential component $A_{y}(t)$ $(-)$ and corresponding electric field $E_{y}(t)(-)$.

$\mathrm{S}_{1}$ state. (It is worth stressing here that this result was obtained with a single trajectory and its only purpose was to compare the two approaches, TSH/LCT and TSH/П-pulse, for a common set of initial conditions.)

In contrast, the TSH/LCT dynamics initialized by using the same conditions applied to the $\Pi$-pulse dynamics gives a smooth and almost complete amplitude transfer from the ground state to the targeted excited state (Figure 3, top panel), with a trajectory hop occurring approximately $10 \mathrm{fs}$ after the maximum of the pulse (when the population of $S_{1}$ is $75.7 \%)$. Interestingly, only a weak population of the two other electronic states $\left(S_{2}\right.$ and $\left.S_{3}\right)$ is observed along the entire dynamics. The Fourier transform (FT) of the LCT pulse (Figure 3, inset of the middle panel) exhibits a distribution of frequencies centered around the GS- $S_{1}$ energy gap $(2.6-2.65 \mathrm{eV}$ with a standard deviation of $\approx 0.3 \mathrm{eV}$ ) with some additional satellite peaks appearing below $2.0 \mathrm{eV}$ that originate from the part of the dynamics evolving on the excited surface (relaxation in $\mathrm{S}_{1}$ ). In fact, the TSH/LCT approach designs a pulse, the frequencies of which closely follow the energy gap between the ground and target states along the dynamics (see below), but it also contains additional features. This complexity of the TSH/LCT frequency spectra relative to that of the one obtained for the $\Pi$-pulse dynamics characterized by a single narrow peak (Figure 2, inset of middle panel) can explain the better performance of the LCT pulse in promoting the population to the target state by using a comparable field strength. At the end of the LCT pulse, the dynamics remains bound to the excited state $S_{1}$. After about $160 \mathrm{fs}$ of dynamics in $S_{1}$, the state amplitudes start to oscillate rapidly due to an increase in the nonadiabatic couplings that accompanies the proton-transfer process (see the discussion below).

Figure 3a-d (bottom) reports some relevant structures of 4HA sampled along the trajectory (labels refer to times indicated in the top panel). The dynamics start in the ground state with the proton linked to the oxygen atom (Figure $3 \mathrm{a}$ ). After 


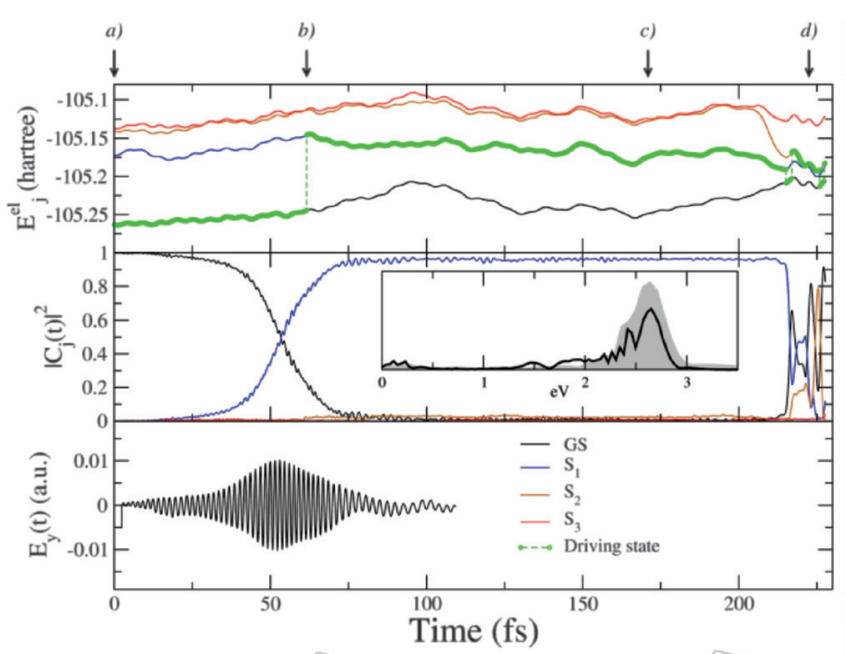<smiles>C=C1C(=C)C2C(=C)C(=O)C3C(=O)C(=C)C(=C)C(=C)C3C2C1=C</smiles>

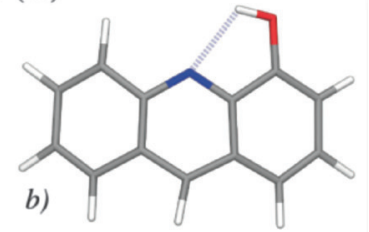

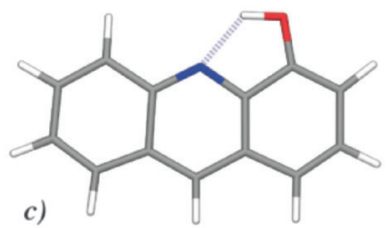<smiles>C=C1C(=C)C(=O)C2C(=O)C(=C)C(=C)C(=O)C2C1=C</smiles>

Figure 3. TSH/LCT dynamics of 4-HA, representative trajectory 1. Top panel) Potential energy curves obtained by using DFT/PBE and LR-TDDFT/PBE/TDA calculations. Color code: GS (black), $S_{1}$ (blue), $S_{2}$ (orange) and $S_{3}$ (red). The green line highlights the force (driving) state. Middle panel) Occupations $\left(\left|C_{j}(t)\right|^{2}\right)$ of all relevant electronic states along the same trajectory. The inset shows the Fourier transforms computed for the entire LCT pulse (-) and for the first part of the pulse until the trajectory hop occurs (light gray area). Bottom panel) Computed local control pulse. Panels (a)-(d) report 4-HA structures sampled along the trajectory (labels refer to times indicated in the top panel).

the surface hop to $S_{1}$ (at $\sim 60 \mathrm{fs}$ ) the $\pi \pi^{*}$ character of the excited state induces a sizeable transfer of electronic density from the oxygen atom (donor) to the nitrogen atom (acceptor). As a consequence, we observe a shortening of the $\mathrm{N}$...H distance and an increase in the $\mathrm{O}-\mathrm{H}$ bond length (Figure $3 \mathrm{c}$ and left panel of Figure 4), which leads to the formation of the product after about $200 \mathrm{fs}$ of dynamics (Figure $3 \mathrm{~d}$ and left panel of Figure 4).

To assess the dependence of the LCT pulse on the initial conditions of the dynamics we ran an additional five trajectories starting from different geometries sampled along the preparative GS Born-Oppenheimer dynamics while keeping all other parameters unchanged. All trajectories reproduce a similar behavior to the one presented above, consisting of smooth population transfer from GS to $S_{1}$ followed by an irreversible surface hop to the excited electronic state $S_{1}$. Interestingly, the profile of the TSH-averaged $S_{1}$ population $\left(\left\langle\left|C_{S_{1}}(t)\right|^{2}\right\rangle_{6}\right)$ and the one of the ensemble trajectory population (corresponding to the percentage of trajectories that at a given time $t$ are driven by the forces of the target state, $S_{1}$ ) agree quite well (Figure 5 ,

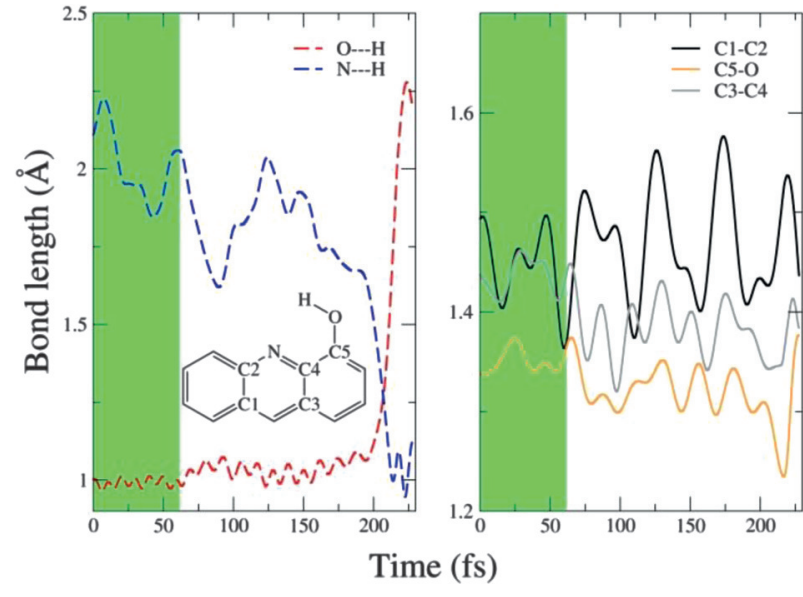

Figure 4. Time evolution of relevant nuclear degrees of freedom of 4-HA along the TSH/LCT dynamics (trajectory 1). The solid green area represents the time window during which the molecule is in its ground state.

inset), which indicates that the TSH dynamics is internally consistent. ${ }^{[50,59]}$ Hops to $S_{3}$ are sometimes observed during the control process, but they are always followed by rapid decay back to the GS. The FTs of the generated LCT pulses (Figure 5) preserve most of the features observed for the first trajectory. The frequency differences can be correlated with the different regions of the molecular configuration space sampled during the pulse-shaping process, whereas the difference in the modulation of the intensities can be linked to the time spent in those regions during the control process. The average of the six frequency spectra preserves the same features observed for the single trajectories, and this confirms the physical nature of the different components, which do not get washed out in the averaging process. Another interesting observation that we gain from the analysis of this ensemble of trajectories is that the proton-transfer process mainly occurs (for five out of six

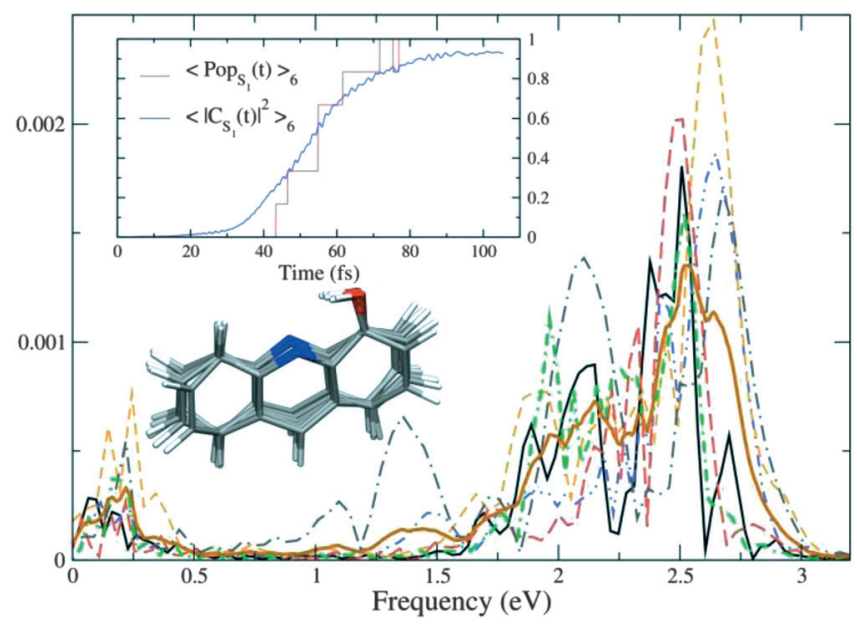

Figure 5. Fourier-transformed TSH/LCT pulses for six trajectories started with different initial conditions (initial structures given in the lower inset). Dark orange line reproduces the average of the six FT spectra. The top inset shows the time evolution of averaged $S_{1}$ state population (computed as the averaged number of trajectories) $\left(\left\langle\operatorname{Pop}_{S_{1}}(t)\right\rangle_{6}\right)$ together with the corresponding averaged probability $\left(\left\langle\left|C_{S_{1}}(t)\right|^{2}\right\rangle_{6}\right)$. 
trajectories) after the control process, at which point the intensity of the applied field is negligibly small. From a more fundamental perspective, it is important to stress that, due to the independent trajectory approximation, LCT within a TSH scheme does not include quantum decoherence effects of the nuclear wavepackets evolving on the same or different electronic states.

More information about the sequence of events that precede and accompany the proton transfer event can be gained from FROG analysis of the pulse frequencies. We applied FROG to the analysis of the dynamics reported in Figure 3: the pulse is initially dominated by a frequency close to the FranckCondon gap (around $2.5 \mathrm{eV}$ ) and undergoes a down-chirp when the trajectory hops into $S_{1}$ (after $\sim 60 \mathrm{fs}$ ) (see Figure 6).

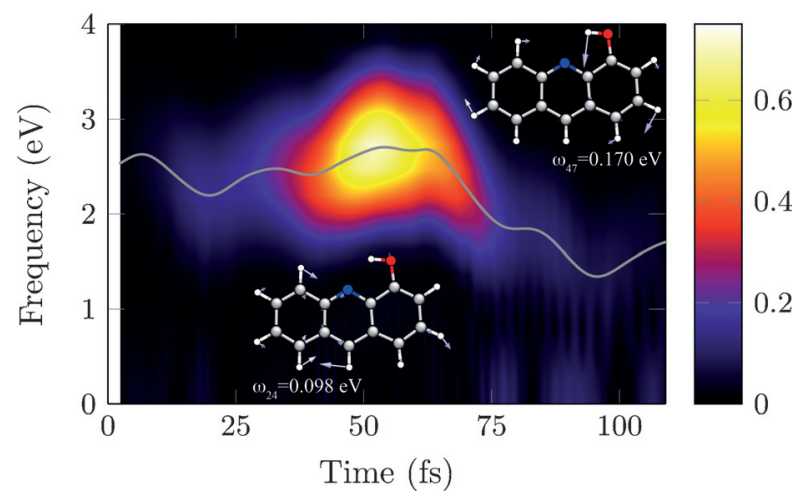

Figure 6. Time-frequency analysis of the control pulse (trajectory 1) calculated by using the frequency-resolved optical grating algorithms; ${ }^{[56,57]}$ it reproduces the time evolution of the LCT pulse spectrum computed in time windows along the trajectory. The gray line depicts the $\left(E_{S_{1}}^{\mathrm{el}}-E_{G S}^{\mathrm{el}}\right)$ gap measured along the same dynamics. Insets: Vector plots of two representative high-frequency modes that contribute to the excited-state dynamics promoting the contraction of the $\mathrm{N}-\mathrm{H}$ distance and the breathing mode of the carbon rings. (The modes were computed at the GS optimized geometry at DFT/PBE/cc-pVDZ level of theory.)

Upon superimposing the time series of the $\left(E_{S_{1}}^{\mathrm{el}}-E_{\mathrm{GS}}^{\mathrm{el}}\right)$ energy gap (gray line in Figure 6) to the FROG trace we observe a clear match of the two time evolutions with the line following the maximum of the spectra at all times. This is once more a clear indication of the nature of the TSH/LCT pulse, which acts in such a way as to guarantee the best population transfer to the target state, as the system evolves in time changing its structure, energy gap, and transition dipole orientation, and which reflects the independent classical trajectory approximation of TSH (see ref. [38] for a discussion on this approximation and its expected consequences in the context of TSH/LCT).

However, there are additional interesting and subtle features that one can obtain from the FROG trace. In particular, we observe the presence of a series of additional weak peaks in the frequency interval between 0.1 and $0.2 \mathrm{eV}$, which become more intense once the trajectory is transferred to the excited state (note that these weak peaks are present in all six trajectories, see Figure 5), contributing in promoting the population transfer to $S_{1}$. These frequencies can be matched with some high-frequency normal modes of the molecular scaffold that, among others, promote the movement of the - $\mathrm{OH}$ group towards the acceptor nitrogen atom (see vector plots in Figure 6). In fact, along the dynamics in $\mathrm{S}_{1}$ we clearly observe significant contraction of the $\mathrm{C} 5-\mathrm{O}$ bond together with activation of the $\mathrm{C} 1-\mathrm{C} 2$ and $\mathrm{C} 3-\mathrm{C} 4$ modes of the aromatic rings (Figure 4, right panel). Once more, it is clear that these components of the FT of the LCT pulse "monitor" the progression of the proton-transfer process in $S_{1}$ rather than drive the reaction itself. Nonetheless, we believe that from the analysis of the FROG trace a good deal can be learned about the detailed mechanisms underlying an intramolecular reaction of this type.

\section{Conclusions}

This work demonstrated the ability of the trajectory surface hopping/local control theory (TSH/LCT) algorithm to control the photoexcited dynamics of a complex molecule without having to limit the sampling of configurational space to few critical modes. In addition, this approach offers the possibility to guide any molecular system to a selected excited state in a more physical way through the action of an explicit time-dependent field, instead of by applying the usual procedure in TSH based on the instantaneous switch of the ground-state forces with those of the target excited state. In particular, the effect of the relative orientation between the transition dipole moments of the compound and the polarization axes of the laser field can be investigated and used in the optimization process.

We also showed that careful analysis of the frequencies contributing to the LCT pulse revealed important and somehow unexpected insight into the complex dynamics underlying the photoinduced proton transfer. The LCT pulse indeed monitored (and did not drive) all molecular vibrations and distortions that took actively part in the process of interest, as revealed from the time dependent analysis of the Fourier transform of the pulse (frequency-resolved optical grating analysis). Of particular interest for the case of the intramolecular proton transfer reaction in 4-hydroxyacridine are all those high-frequency vibrational modes of the molecular scaffold that favor the approaching of the proton donor and acceptor groups. Investigations performed on low-dimension model systems of the same compound (e.g. by using constraint dynamics) are likely to miss the contribution of some of these collective modes that are difficult to predict a priori.

The method proposed in this study is, however, still in its infancy and more theoretical and numerical studies are necessary to access some pending issues. For instance, the influence of the initial conditions on the characteristics of the designed pulse should still be carefully addressed as well as the effects related to the quantum mechanical and statistical average over an ensemble of trajectories. In fact, while a single-molecule pulse obtained for a given TSH trajectory is able to efficiently transfer population from the ground state to a desired electronic state (triggering the reaction of interest), its effect on the ensemble population is not guaranteed. However, by using a limited sampling of trajectories (six in total), we were able to show that some of the most relevant features of the 
frequency spectra are common to all trajectories, which therefore suggests a possible way to construct an effective pulse for an ensemble of molecules.

Finally, further studies are needed to shed light on the effects of nuclear wavepacket decoherence in the pulse-shaping process, ${ }^{[50]}$ which are still neglected in the standard TSH approaches due to the independent trajectory approximation. ${ }^{[38,60]}$

\section{Acknowledgements}

I.T. acknowledges support and finance through the Swiss National Science Foundation (grant SNF SER-C09.0046) and the COSTaction CM0702. U.R. acknowledges the NCCR MUST for support.

Keywords: density functional calculations - local control theory $\cdot$ molecular dynamics - photophysics $\cdot$ proton transfer

[1] R. Judson, H. Rabitz, Phys. Rev. Lett. 1992, 68, 1500.

[2] H. Rabitz, R. de Vivie-Riedle, M. Motzkus, K. Kompa, Science 2000, 288, 824.

[3] R. Levis, G. Menkir, H. Rabitz, Science 2001, 292, 709.

[4] P. W. Brumer, M. Shapiro, Principles of the Quantum Control of Molecular Processes, Wiley, Hoboken, NJ, 2003.

[5] W. S. Warren, H. Rabitz, M. Dahleh, Science 1993, 259, 1581

[6] A. Assion, T. Baumert, M. Bergt, B. Briner, T. Kiefer, V. Seyfried, M. Sterile, G. Gerber, Science 1998, 282, 919.

[7] A. P. Peirce, M. A. Dahleh, H. Rabitz, Phys. Rev. A 1988, 37, 4950.

[8] H. Katsuki, H. Chiba, C. Meier, B. Girard, K. Ohmori, Phys. Rev. Lett. 2009, $102,103602$.

[9] Y. Okano, H. Katsuki, Y. Nakagawa, H. Takahashi, K. G. Nakamura, K. Ohmori, Faraday Discuss. 2011, 153, 375

[10] J. Herek, W. Wohlleben, R. Cogdell, D. Zeidler, M. Motzkus, Nature 2002, $417,533$.

[11] G. Vogt, G. Krampert, P. Niklaus, P. Nuernberger, G. Gerber, Phys. Rev. Lett. 2005, 94, 068305.

[12] V. I. Prokhorenko, A. M. Nagy, S. A. Waschuk, L. S. Brown, R. R. Birge, R. J. D. Miller, Science 2006, 313, 1257.

[13] T. Brixner, N. H. Damrauer, P. Niklaus, G. Gerber, Nature 2001, 414, 57.

[14] M. Roth, L. Guyon, J. Roslund, V. Boutou, F. Courvoisier, J.-P. Wolf, H. Rabitz, Phys. Rev. Lett. 2009, 102, 253001.

[15] J. Petersen, R. Mitric, V. Bonacic-Koutecky, J. Wolf, J. Roslund, H. Rabitz, Phys. Rev. Lett. 2010, 105, 73003.

[16] P. Nuernberger, D. Wolpert, H. Weiss, G. Gerber, Proc. Natl. Acad. Sci. USA 2010, 107, 10366

[17] Y. Silberberg, Annu. Rev. Phys. Chem. 2009, 60, 277.

[18] V. Prokhorenko, A. Halpin, R. Miller, Faraday Discuss. 2011, 153, 27.

[19] E. Goulielmakis, V. S. Yakovlev, A. L. Cavalieri, M. Uiberacker, V. Pervak, A. Apolonski, R. Kienberger, U. Kleineberg, F. Krausz, Science 2007, 317, 769.

[20] D. J. Tannor, S. A. Rice, J. Chem. Phys. 1985, 83, 5013.

[21] P. Brumer, M. Shapiro, Chem. Phys. Lett. 1986, 126, 541.

[22] R. Kosloff, S. Rice, P. Gaspard, S. Tersigni, D. Tannor, Chem. Phys. 1989, 139, 201.

[23] C. Daniel, J. Full, L. González, C. Lupulescu, J. Manz, A. Merli, Š. Vajda, L. Wöste, Science 2003, 299, 536.
[24] J. Werschnik, E. Gross, J. Phys. B 2007, 40, R175.

[25] M. Thachuk, M. Y. Ivanov, D. M. Wardlaw, J. Chem. Phys. 1996, 105, 4094.

[26] K. Yagi, K. Takatsuka, J. Chem. Phys. 2005, 123, 224103.

[27] J. C. Tully, J. Chem. Phys. 1990, 93, 1061.

[28] T. Vreven, F. Bernardi, M. Garavelli, M. Olivucci, M. Robb, H. Schlegel, J. Am. Chem. Soc. 1997, 119, 12687.

[29] M. Barbatti, M. Vazdar, A. J. A. Aquino, M. Eckert-Maksic, H. Lischka, J. Chem. Phys. 2006, 125, 164323.

[30] G. Granucci, M. Persico, A. Toniolo, J. Chem. Phys. 2001, 114, 10608.

[31] E. Fabiano, T. Keal, W. Thiel, Chem. Phys. 2008, 349, 334

[32] E. Tapavicza, I. Tavernelli, U. Rothlisberger, Phys. Rev. Lett. 2007, 98, 023001.

[33] R. Mitric, U. Werner, V. Bonacic-Koutecký, J. Chem. Phys. 2008, 129, 164118.

[34] G. A. Jones, A. Acocella, F. Zerbetto, J. Phys. Chem. A 2008, 112, 9650.

[35] R. Mitric, J. Petersen, V. Bonacic-Koutecký, Phys. Rev. A 2009, 79, 053416.

[36] M. Richter, P. Marquetand, J. González-Vázquez, I. Sola, L. González, J. Chem. Theory Comput. 2011, 7, 1253.

[37] I. Tavernelli, B. F. E. Curchod, U. Rothlisberger, Phys. Rev. A 2010, 81, 052508.

[38] B. F. E. Curchod, T. J. Penfold, U. Rothlisberger, I. Tavernelli, Phys. Rev. A 2011, 84, 042507.

[39] D. Tannor, R. Kosloff, A. Bartana, Faraday Discuss. 1999, 113, 365.

[40] V. Engel, C. Meier, D. J. Tannor, Adv. Chem. Phys. 2009, 141, 29.

[41] T. J. Penfold, G. A. Worth, C. Meier, Phys. Chem. Chem. Phys. 2010, 12, 15616.

[42] R. Singh, S. Mahanta, N. Guchhait, J. Photochem. Photobiol. A 2008, 200, 325.

[43] B. Paul, S. Mahanta, R. Singh, N. Guchhait, J. Phys. Chem. A 2010, 114, 2618.

[44] B. F. E. Curchod, U. Rothlisberger, I. Tavernelli, ChemPhysChem 2013, 14, 1314.

[45] E. Tapavicza, G. D. Bellchambers, J. C. Vincent, F. Furche, Phys. Chem. Chem. Phys. 2013, 15, 18336.

[46] P. Marquetand, V. Engel, Chem. Phys. Lett. 2005, 407, 471.

[47] L. Bomble, A. Chenel, C. Meier, M. Desouter-Lecomte, J. Chem. Phys. 2011, 134, 204112.

[48] L. González, P. Marquetand, M. Richter, J. González-Vázquez, I. Sola, Springer Ser. Chem. Phys. 2014, 107, 145.

[49] B. F. E. Curchod, I. Tavernelli, J. Chem. Phys. 2013, 138, 184112.

[50] J. J. Bajo, G. Granucci, M. Persico, J. Chem. Phys. 2014, 140, 044113.

[51] CPMD, Version 3.15 (Copyright IBM Corp. 1990-2008, Copyright MPI für Festkörperforschung Stuttgart 1997-2001, 1990-2008), http:// www.cpmd.org.

[52] N. Troullier, J. L. Martins, Phys. Rev. B 1991, 43, 1993.

[53] J. Hutter, J. Chem. Phys. 2003, 118, 3928.

[54] J. P. Perdew, K. Burke, M. Ernzerhof, Phys. Rev. Lett. 1996, 77, 3865.

[55] C. Adamo, V. Barone, J. Chem. Phys. 1999, 110, 6158.

[56] G. A. Worth, M. H. Beck, A. Jäckle, H.-D. Meyer, The Heidelberg MCTDH Package: A Set of Programs for Multidimensional Quantum Dynamics. User's Guide, Version 8, 2000 (The User's Guide can be downloaded from the URL: http://www.pci.uni-heidelberg.de/tc/usr/mctdh/).

[57] S. Linden, H. Giessen, J. Kuhl, Phys. Status Solidi B 1998, 206, 119, ISSN: 1521-3951.

[58] W. Humphrey, A. Dalke, K. Schulten, J. Mol. Graphics 1996, 14, 33.

[59] G. Granucci, M. Persico, J. Chem. Phys. 2007, 126, 134114

[60] B. F. E. Curchod, T. J. Penfold, U. Rothlisberger, I. Tavernelli, Chimia 2013, $67,218$.

Received: March 3, 2015

Published online on June 3, 2015 